\title{
Repeatedly heated mix vegetable oils- induced atherosclerosis and effects of Murraya koenigii
}

\author{
Gul Ambreen ${ }^{1,2^{*}}$ D, Afshan Siddiq ${ }^{1}$, Kashif Hussain², Abdul Saboor Hussain ${ }^{1}$ and Zara Naz ${ }^{3}$
}

\begin{abstract}
Background: Statins are considered as standard drugs to control cholesterol levels, but their use is also associated with renal hypertrophy, hemorrhagic stroke, hepatomegaly, and myopathy. Murraya koenigii is an herb that is used in traditional cuisine and as a medicine in South Asia. Here we assessed the antidyslipidemic and antiatherosclerotic effects of this spice in repeated heated mix vegetable oils (RHMVO)-induced atherosclerotic models.

Methods: Aqueous extract of M. koenigii leaves (Mk LE) was prepared and its phytoconstituents were determined. Rabbits were divided into 5 groups $(n=10)$. Except for the control group, all the other four groups were treated with RHMVO for 16 weeks (dose $=2 \mathrm{ml} / \mathrm{kg} /$ day) to induce dyslipidemia and atherosclerosis. These groups were further treated for 10 weeks either with 300 and 500 mg/kg/day Mk LE, lovastatin, RHMVO, or left untreated. Body and organ weights were measured along with oxidative stress and tissue damage parameters. Lipid profile and hepatic function markers were studied. Atheroma measurement and histopathological examination were also performed in control and treated groups.
\end{abstract}

Results: Mk LE significantly $(p<0.05)$ attenuated RHMVO-induced dyslipidemia and atheroma formation. Furthermore, fat accumulation and lipid peroxidation in hepatic tissues were reduced by Mk LE in a dosedependent manner. Our results indicated that the antidyslipidemic effects of Mk LE in $500 \mathrm{mg} / \mathrm{kg} /$ day dose were comparable to lovastatin. Additionally, oxidative stress markers were reduced much more significantly in Mk LE-500 than in the statin group $(p<0.05)$.

Conclusions: This study recommends Mk LE as a potent antioxidant and lipid-lowering natural medicine that can attenuate the RHMVO-induced atherosclerotic in optimal doses and duration. Therefore, Mk LE can be accessible, cheap, and free of adverse effects alternate to statins.

Keywords: Murraya koenigii, Repeated heated, Vegetable oils, Oxidative stress

\footnotetext{
* Correspondence: gul.ambreen@aku.edu

'Department of Pharmacology, Faculty of Pharmacy and Pharmaceutical

Sciences, University of Karachi, Karachi, Pakistan

${ }^{2}$ Department of Pharmacy, Aga Khan University Hospital, Stadium Road (Main

Pharmacy), P.O Box 3500, Karachi 74800, Pakistan

Full list of author information is available at the end of the article
}

(c) The Author(s). 2020 Open Access This article is licensed under a Creative Commons Attribution 4.0 International License, which permits use, sharing, adaptation, distribution and reproduction in any medium or format, as long as you give appropriate credit to the original author(s) and the source, provide a link to the Creative Commons licence, and indicate if changes were made. The images or other third party material in this article are included in the article's Creative Commons licence, unless indicated otherwise in a credit line to the material. If material is not included in the article's Creative Commons licence and your intended use is not permitted by statutory regulation or exceeds the permitted use, you will need to obtain permission directly from the copyright holder. To view a copy of this licence, visit http://creativecommons.org/licenses/by/4.0/ The Creative Commons Public Domain Dedication waiver (http://creativecommons.org/publicdomain/zero/1.0/) applies to the data made available in this article, unless otherwise stated in a credit line to the data. 


\section{Background}

In modern society, altered food consumption pattern increases incidences of metabolic syndrome, including obesity, hypertension, diabetes, atherosclerosis, stroke, and cancer [1-4]. Particularly dyslipidemia is considered as the basic cause of health concerns, related to metabolic syndrome [2]. As per National Cholesterol Education Program Adult Treatment Panel III (NCEP-ATP) guidelines, dyslipidemia is characterized as triglycerides (TG) $>150 \mathrm{mg} / \mathrm{dl}$, total serum cholesterol $(\mathrm{TC})>200 \mathrm{mg} / \mathrm{dl}$, low-density lipoproteins (LDL) $>100 \mathrm{mg} / \mathrm{dl}$ and high-density lipoproteins (HDL) less than $40 \mathrm{mg} / \mathrm{dl}$. Dyslipidemia is the most important risk factors of cardiovascular diseases (CVDs) and atherosclerosis [5, 6].

Cholesterol is transported to the liver for metabolism, by HDL, whereas endothelial injuries and formation of atheroma are triggered by oxidized LDL molecule. Therefore, reduction of LDL and TC, along with HDL level enhancement is targeted to prevent atherosclerosis [7]. So far, statins have been utilized for inhibiting cholesterol biosynthesis, by restraining 3-hydroxy-3methylglutary-coenzyme A (HMG-CoA). In addition, statins induce the cellular level of LDL receptors activity that in turn facilitates the metabolism of LDL and verylow-density lipoproteins (VLDL) [8, 9]. However, despite this benefit, long-term use, and/or over-dosage of statins results in serious adverse effects, such as renal hypertrophy, hemorrhagic stroke, hepatomegaly, and myopathy [10-12]. These facts are driving the researchers to explore the solution from nature to regulate blood flow and control the blood cholesterol levels, with the least adverse effects.

Physico-chemical characteristics of the cooking oils change when they are heated beyond a certain limit as several chemical reactions take place in the presence of moisture and air. Oils degenerate and produce volatile substances, unwanted monomers, polymers, isomers, and free radicals [13]. In cooking oil fatty acids (FAs) naturally exist in the cis-isomer form, but during thermal oxidation they convert into trans-isomers, possessing physical properties similar to saturated FAs [14]. Several animal studies demonstrate that consumption of thermally oxidized vegetable oils increased the risk of CVDs, like hypertension with reduced vasorelaxation responses [15], endothelial malfunction [16], lipid peroxidation [17], atherosclerosis [18], and oxidative stress [19]. However, regardless of all these studies, the practice of reusing and overheating the cooking oils while processing the food seems to continue.

$M$. koenigii (curry leaves) are commonly used spices in Pakistani cuisine. With the vast range of biological activities such as antidyslipidemic [20-22], platelet aggregation inhibiting [23], antioxidant [24], and hypoglycemic activities [25], it gained the attention of researchers worldwide. Several animal studies have reported this spice as a potent antidyslipidemic agent and the response was achieved in 4-8 weeks, in a dose-dependent manner [20, 21]. A recent human study, conducted on 45-65 years women of postmenopausal phase with hyperlipidemia, also reported that $M$. koenigii leaves intake for 45 consecutive days can significantly reduce the blood lipid levels [22]. A study also reported that Mk LE inhibits platelet aggregation, resulting in improved blood flow [23]. It also acts as an antioxidant, further suppressing the vascular inflammatory response through reduced reactive oxygen species (ROS) production [26].

Presently, several animal models have been used to study hyperlipidemia induced atherosclerosis, like mice [27], zebrafish [28], rats [29, 30], and pigs [31]. To study the effect of curry leaves on thermally oxidized oil-induced hypercholesterolemia and atherosclerosis selection of experimental animal models is very critical to conduct basic research and develop a study tool. In this respect, to study human hyperlipidemia the rabbit has become the most appropriate animal model because of rabbits' unique features of lipid metabolism similar to humans [32,33]. Several studies also used rabbit model for cardiac diseases [34]. Rabbit as an experimental animal is also considered appropriate for the Rabbit as an experimental animal model is also used to evaluate the medicinal effects of several herbal medicines, like the hypolipidemic effect of Ficus bengalensis [35], the hypoglycemic effect of Achyranthes aspera [36] antihyperglycemic effects of Alpinia galanga rhizome and its extracts [37], and hypoglycemic effects of $M$. koenigii [25].

In Pakistan, commercially available oils are mostly a blend of two or more edible oils, and the most common available blend of an equal ratio of olive, canola, and sunflower oils was focused in this study, to have the model that mimics the human situation. Vegetable oils are repeatedly heated when used for food processing (frying) at household and commercial levels. We used repeatedly heated mixed vegetable oils (RHMVO) to induce hyperlipidemia and atherosclerosis. We designed this study to investigate the medicinal effects of curry leaves in different doses, in RHMVO-induced atherosclerotic models.

\section{Methods}

Collection and identification of oils and plant material

Fresh curry leaves, standard food-grade canola, sunflower, and olive oils were purchased from Karachi local market. The specimen of plant material and oils were verified by the Department of Pharmacognosy, University of Karachi. 


\section{Oil sample preparation}

Olive, canola, and sunflower oils were mixed in equal ratio and heated beyond the smoke point of the oil mixture at $220^{\circ} \mathrm{C}$ for $45 \mathrm{~min}$ each day for a total of ten days [38-40]. The smoke point for olive oil, canola oil, sunflower oil is $180{ }^{\circ} \mathrm{C}, 200^{\circ} \mathrm{C}$, and $225^{\circ} \mathrm{C}$, respectively [41, 42]. Every day before heating, the oil level was adjusted in the pan with the same oils blend $(0.007 \pm 0.0031$ per day) to meet the initial level. This replenishment method was adopted to replicate the same practices used in fried and fast food outlets [38]. At the end of the 10th day, viscous dark brown oil was obtained. To prevent photodegradation it was stored in amber color bottles and labeled as RHMVO [43].

\section{Preparation of Murraya koenigii leaves extract (Mk LE)}

Curry leaves were cleaned by thorough washing to remove any contamination with double-distilled water. Washed curry leaves were dried in shade at room temperature and grounded to powder with the help of a mechanical grinder and then preserved at room temperature in an airtight container. Three hundred grams powdered leaves were subjected to cold extraction by stirring them in 4-1 double-distilled water for $24 \mathrm{~h}$. The resultant suspension was centrifuged at $10,000 \times \mathrm{g}$ for $10 \mathrm{~min}$ and filtered through Whatman No. I filter paper, followed by a $0.45 \mu \mathrm{m}$ membrane filter (Sartorius minisart, Hannover, Germany). Finally, the filtrate was dried using a rotary evaporator and resulted in a $13.6 \%$ yield. The dried extract was dissolved in distilled water to achieve $100 \mathrm{mg} / \mathrm{ml}$ stock. Crude aqueous extract of curry leaves (Mk LE) was then kept at $4{ }^{\circ} \mathrm{C}$ storage temperature for subsequent use for planned experiments.

\section{Experimental animals}

Healthy adult male rabbits of local strain weighing between $1450 \pm 10 \mathrm{~g}$ were purchased from the department of pharmacology, University of Karachi, Pakistan. Male rabbits were selected to avoid the sex difference due to female sex hormonal effects on CVDs $[44,45]$. Secondly, the prevalence of plasma lipid abnormalities in Pakistan, are higher in males than females [46, 47]. Rabbits were kept individually in wire-topped steel cages with a wooden bottom, under controlled humidity (50-60\%), and temperature $\left(23^{\circ} \mathrm{C} \pm{ }^{\circ} \mathrm{C}\right)$ with $12 / 12 \mathrm{~h}$ light/dark photo-cycle. Animals were acclimatized for 7 days before starting the experiment. Animals were handled according to the institutional animal's ethical committee guidelines.

\section{Experimental design}

The total experimental period was of 26 weeks and all animals were divided into five groups $(n=10)$. In the first 16 weeks, except for the control, all other groups were treated with $2 \mathrm{ml} / \mathrm{kg} /$ day of RHMVO to induce hypercholesterolemia [43]. The induction period was followed by the treatment period of 10 weeks. In the treatment period animals were treated as follows

- Control group: Untreated

- RHMVO group: Rabbits continued to have $2 \mathrm{ml} / \mathrm{kg} /$ day RHMVO

- Mk LE-300 group: Rabbits were given Mk LE 300 $\mathrm{mg} / \mathrm{kg} /$ day

- Mk LE-500 group: Rabbits were given Mk LE 500 $\mathrm{mg} / \mathrm{kg} /$ day

- Statin group: Rabbits were given lovastatin $0.5 \mathrm{mg} /$ $\mathrm{kg} /$ day

From the statin group, we selected lovastatin, to avoid the additional side effects (nasopharyngitis, diarrhea, and urinary tract infection) which are associated with other statins [48]. Oil and Mk LE were administered daily in the morning and lovastatin in the evening through the oral route, with the help of oral syringes [49]. Lovastatin, a short-acting statin, is prescribed to be taken in the evening to achieve better results as cholesterol production through livers enzymes is higher in these hours [50, 51]. For the rest of the day animals of all the groups were fed ad libitum on a regular normal diet (fresh hay and water). Morbidity and mortality were monitored through the study period. Animal's body weight was recorded at baseline, week 1, 16, 20, 23, and 26. After the induction period (at 16 weeks) and treatment period (at 26 weeks), 3 rabbits from each group were euthanized through the intravenous administration of sodium pentobarbital $100 \mathrm{mg} / \mathrm{kg}$ followed by decapitation [52]. Furthermore, animals were necropsied for histopathological examination of internal organs and to retrieve liver, heart, spleen and kidney for organs weight evaluation.

\section{Blood sample collection for lipid profile and liver function markers}

Blood samples were collected from the ear vein of animals at week 1, 16, 20,23, and 26. For hematological parameters, $2 \mathrm{ml}$ blood was collected in EDTA and 5 $\mathrm{ml}$ in gel vacutainers to perform the biochemical assays. Blood was centrifuged to collect plasma and serum and stored at $-20^{\circ} \mathrm{C}$ until used further to analyze the plasma lipid profile (TG, TC, HDL, LDL, VLDL) and liver function markers, such as total protein, albumin, serum glutamic-pyruvic transaminase (SGPT), serum glutamic-oxaloacetic transaminase (SGOT) and alkaline phosphatase (ALP) using commercially available kits (RANDOX Laboratories Ltd) followed by manufacturer's instructions. 


\section{Oxidative stress and tissue damage parameters}

Lipid peroxidation was measured by determining plasma malondialdehyde (MDA), following Kikugawa et al method [53]. The plasma C-reactive protein (CRP) was measured by using an ELISA kit (Abnova, Taipei, Taiwan), as per the manufacturer's instruction. A commercially available kit was used to analyses lactate dehydrogenase (LDH) (BioVision, USA). We followed Hamsi et al. method to prepare the samples and standards [54]. Sheu et al. method was adopted to measures the fasting plasma homocysteine concentrations [55]. Creatine phosphokinase (CPK) was measured by a commercially available kit from Beckman Coulter, Brea, USA, following manufacturer instructions.

\section{Atheroma measurement}

After 26 weeks animals were sacrificed as per the guidelines and necropsied to dissect a $10 \mathrm{~cm}$ aortic arch from the aortic valve and washed in normal saline followed by further dissection to open the orifice of the carotid artery ( $5 \mathrm{~cm}$ long) with a longitudinal cut. After removal of adhered tissue, propylene glycol (100\%) was used to dehydrate the aorta, for $10 \mathrm{~min}$ followed by another $10 \mathrm{~min}$ staining $0.7 \%$ Sudan IV (in propylene glycol) [56-58]. The tissue was re-hydrated by treating it with $85 \%$ propylene glycol for $5 \mathrm{~min}$, washed with distilled water, and photographed. Digital Image Analyzer was used to analyze the red atheromatous plaques. We measured the degree of lipid deposition by calculating the percent atherosclerosis index (AI) through the ratio between the Sudan-positive area to the whole aortic wall area.

\section{Microscopic examination}

After weighing liver tissues, samples were treated with alcohol to dehydrate. After cleaning with xylene, samples were further embedded in paraffin at $56{ }^{\circ} \mathrm{C}$. By using a rotatory microtome, $5 \mu \mathrm{m}$ sized sections were obtained, mounted onto albumenized slides, and kept for drying for $12 \mathrm{~h}$. Dewaxing was done in xylene and hydration was performed in ethanol and water. The sections were first treated with Harris hematoxylin to stain, thereafter differentiation by acid alcohol, and then staining in methylene blue was performed. Furthermore, dehydration of sections in alcohol (95\%) and staining in alcoholic eosin (10\%), dehydration with absolute alcohol, and cleaning with xylene were completed. The sections were then mounted using Canada balsam. These prepared slides were finally viewed with the use of a light microscope.

\section{Phytoconstituents determination of Mk LE}

For the quantitative measurement of the total content of flavonoids and phenols in the Mk LE, we followed the methods of Sefi et al [59]. Total phenol content is mentioned in mg gallic acid equivalents (GAE) per each gram of extract, where GA was considered as standard. Total flavonoids are mentioned as mg catechin equivalents (CE) per each gram of extract, catechin was used as standard. For the quantitative finding of the total alkaloid content in the Mk LE, we followed the method of Sreevidya et al [60] and expressed as $\mathrm{mg} / \mathrm{g}$ bismuth nitrate. Total tannin content was evaluated by following the Polshettiwar et al method [61] and expressed as tannic acid equivalent per gram of Mk LE. Total chlorophyll content was evaluated by following the method adopted in Kizhedath and Suneetha study [62] and expressed as mg pigments/gm.

\section{Statistical analysis}

Data is represented in the form of mean \pm standard deviation. We applied analysis of variance (ANOVA) followed by post hoc Tukey's honest significant difference (HSD) test to find out statistical significance at $p<$ 0.05 . Statistical product and service solutions software was used.

\section{Results}

\section{Biometric parameters}

No death and unexpected signs and symptoms noticed in experimental animals throughout the study period in the treated animals and control. The body and organ weights of animals in all the groups increased gradually with age (Table 1 and Fig. 1). Bodyweight of all the animals treated with RHMVO was significantly higher than control, after the induction period of 16 weeks. At 26 weeks animals in the RHMVO group exhibited significantly higher weight than the age-matched other groups. However, statistically insignificant differences were present in the bodyweight of Mk LE-300, Mk LE-500 and lovastatin groups at 20th week, but at the end of 26th week, this phenomenon became very considerable and statistically significant body weight reduction was observed in Mk LE treated groups in a dose-dependent manner compared to RHMVO fed group $(p<0.05)$. Moreover, weight reduction was also statistically significant in the lovastatin treated group $(p<0.05)$, but lesser than the Mk LE-500 group. As shown in Table-1 organs weight reduction of Mk LE treated groups in the treatment phase was dose-dependent and statistically highly significant $(p<0.005)$ in $500 \mathrm{mg} / \mathrm{kg} /$ day doses. Organ weight reduction in Mk LE-500 treated group was even higher than the lovastatin treated group.

\section{Serum biochemical analysis of lipid profile}

As shown in Table 2, animals in RHMVO exhibited higher TGs, TC, LDL, VLDL, and lower HDL circulating levels in comparison with control. After 26 weeks of RHMVO feeding the TGs and TC values increased 
Table 1 Comparison of organs weight post-induction and after-treatment

\begin{tabular}{lllllll}
\hline Organs & Observation Time & Control (weight-g) & RHMVO (weight-g) & Mk LE-300 (weight-g) & Mk LE-500 (weight-g) & Lovastatin (weight-g) \\
\hline Liver & Post induction (week16) & $53.66 \pm 1.35$ & $86.25 \pm 2.62$ & $87.38 \pm 1.88$ & $85.26 \pm 1.92$ & $86.24 \pm 2.22$ \\
& After treatment (week26) & $60.31 \pm 4.33$ & $104.95 \pm 2.6 \# \#$ & $83.76 \pm 1.89$ & $63.17 \pm 2.56^{* *}$ & $75.51 \pm 2.26^{*}$ \\
\multirow{2}{*}{ Spleen } & Post induction (week16) & $34.37 \pm 1.47$ & $56.89 \pm 2.15$ & $57.1 \pm 2.25$ & $55.75 \pm 3.09$ & $55.09 \pm 3.46$ \\
& After treatment (week26) & $40.88 \pm 3.42$ & $74.96 \pm 4.5 \#$ & $54.08 \pm 2.26$ & $42.48 \pm 1.2^{* *}$ & $51.27 \pm 2.52$ \\
Heart & Post induction (week16) & $18.08 \pm 1.39$ & $34.75 \pm 0.9$ & $34.92 \pm 1.09$ & $34.9 \pm 1.14$ & $34.95 \pm 1.15$ \\
& After treatment (week26) & $18.54 \pm 5.92$ & $49.17 \pm 4.84 \#$ & $32.83 \pm 1.09$ & $29.31 \pm 1.14^{*}$ & $31.86 \pm 1.12$ \\
Kidney & Post induction (week16) & $14.41 \pm 0.9$ & $27.74 \pm 1.53$ & $27.97 \pm 1.63$ & $27.77 \pm 1.74$ & $28.11 \pm 1.94$ \\
& After treatment (week26) & $17.98 \pm 2.43$ & $37.16 \pm 2.18 \# \#$ & $25.99 \pm 1.28$ & $21.05 \pm 1.38^{*}$ & $25.6 \pm 0.55$ \\
\hline
\end{tabular}

*Significantly reduced from post-induction weight $(p<0.05)$

**Significantly reduced from post-induction weight $(p<0.005)$

\# significantly increased from the post-induction weight $(p<0.05)$

\#\# Significantly increased from post-induction weight $(p<0.005)$

about 4-5 folds in the RHMVO group. Whereas in Mk LE treated groups all these parameters improved in a dose-dependent manner and statistically significant $(p>0.05)$ changes were observed in Mk LE-500 and lovastatin groups. Although, at weeks 20 and 23 there was no such obvious difference between Mk LE-300, Mk LE500 , and statin groups. But at the end of 26 weeks Mk LE-500 group animals presented significantly $(p>0.005)$ improved lipid profile than Mk LE-300 and comparable to statin group. At the end of treatment HDL levels were significantly higher in the Mk LE-500 group compared to the control group.

\section{Hepatic function and oxidative stress markers}

The biochemical analysis of liver function markers has shown that continuous feeding of RHMVO induced hypercholesterolemia, which resulted in significantly $(p<0.005)$ reduced total protein and albumin levels. The blood levels of LDH, CPK, SGOT, SGPT, and ALP were also increased significantly, suggestive of liver

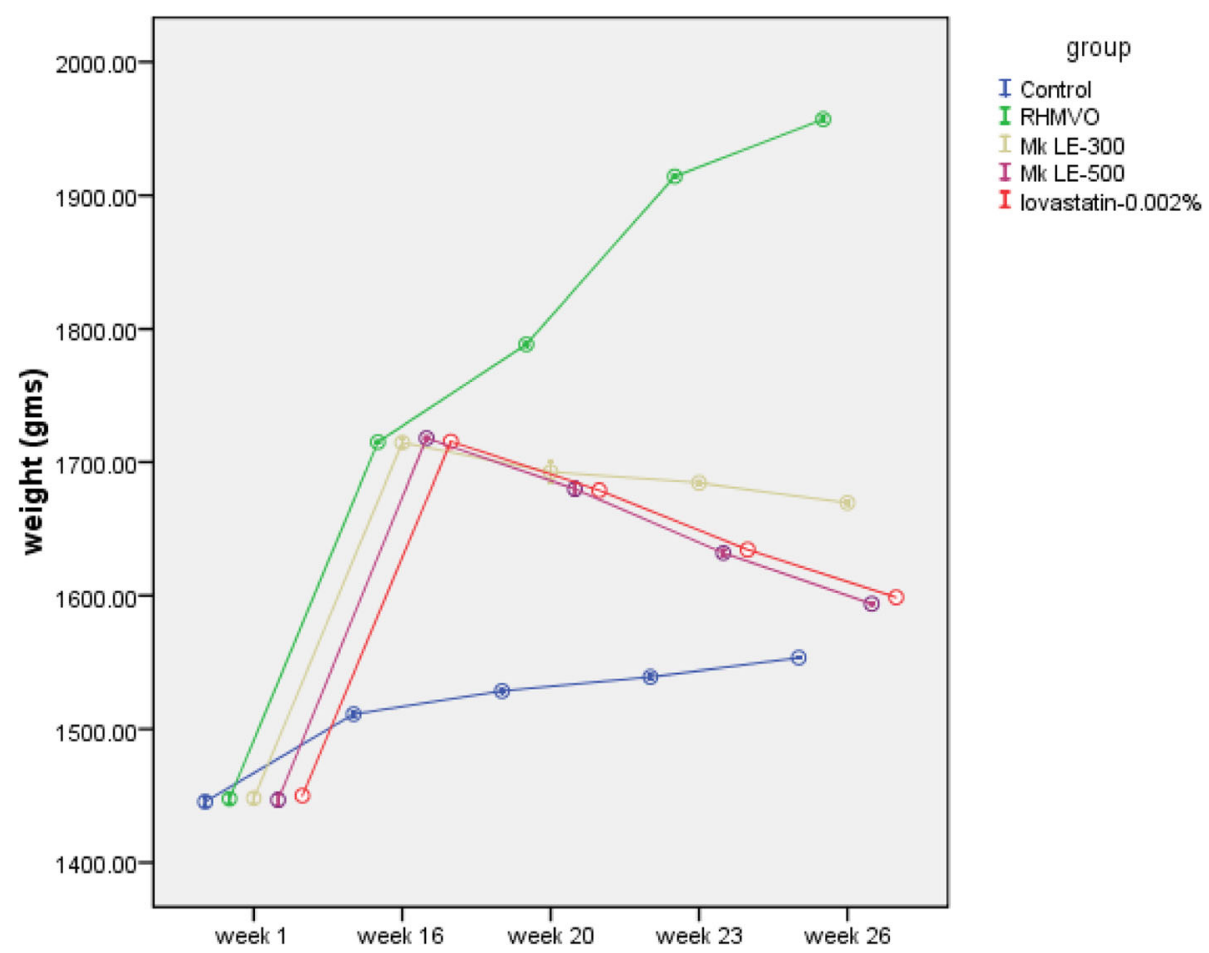

Fig. 1 Graphical Presentation, Comparing the Weight Gain in Different Groups During the Study Period (at 95\% Cl): (Control = Blue line showing the gradual increment in bodyweight with age, RHMVO fed group = Green line showing significant weight gain, Mk LE-300 group = Brown line showing the weight reduction over week 16 to 26 , lovastatin group = Red line showing significant weight reduction, Mk LE-500 group= Purple line showing the significant weight reduction comparable with red line of lovastatin group 
Table 2 Comparison of lipid profile through the study period (baseline till week-26)

\begin{tabular}{|c|c|c|c|c|c|}
\hline Observation Time & Control & RHMVO & Mk LE-300 & Mk LE-500 & Lovastatin \\
\hline \multicolumn{6}{|l|}{ TRIG (mg/dl) } \\
\hline week 1-baseline (Before induction) & $56.32 \pm 3.51$ & $55.43 \pm 2.21$ & $55.62 \pm 3.28$ & $57.08 \pm 3.80$ & $56.58 \pm 3.18$ \\
\hline week 16 (Post induction) & $69.08 \pm 2.92$ & $272.18 \pm 1.76^{* *}$ & $279.61 \pm 6.76^{* *}$ & $281.4 \pm 6.03^{* *}$ & $276.01 \pm 12.31^{* *}$ \\
\hline week 20 & $73.17 \pm 2.11$ & $309.26 \pm 1.83^{* *}$ & $251.83 \pm 6.16$ & $256.01 \pm 6.19$ & $255.67 \pm 12.05$ \\
\hline week 23 & $74.75 \pm 3.42$ & $330.61 \pm 1.55^{* *}$ & $220.05 \pm 6.28$ & $218.76 \pm 5.05$ & $224.46 \pm 12.92$ \\
\hline week 26 & $75.93 \pm 4.0$ & $352.07 \pm 6.74^{* *}$ & $179.06 \pm 6.09 \#$ & $166.51 \pm 3.08 \# \#$ & $195.2 \pm 12.41 \#$ \\
\hline \multicolumn{6}{|l|}{$\mathrm{TC}(\mathrm{mg} / \mathrm{dl})$} \\
\hline week 1- baseline (Before induction) & $141.02 \pm 3.21$ & $140.12 \pm 3.25$ & $141.21 \pm 5.02$ & $141.30 \pm 3.55$ & $141.25 \pm 1.58$ \\
\hline week 16 (Post induction) & $148.54 \pm 4.25$ & $444.5 \pm 8.23^{* *}$ & $446.31 \pm 8.59^{* *}$ & $450.35 \pm 8.91^{* *}$ & $443.29 \pm 11.61^{* *}$ \\
\hline week 20 & $150.19 \pm 5.23$ & $503.45 \pm 8.09^{* *}$ & $394.07 \pm 8.23$ & $378.1 \pm 8.09$ & $388.08 \pm 11.06$ \\
\hline week 23 & $151.84 \pm 3.02$ & $562.4 \pm 8.55^{* *}$ & $350.75 \pm 7.99$ & $289.85 \pm 8.23 \#$ & $333.82 \pm 11.31$ \\
\hline week 26 & $154.4 \pm 3.87$ & $637.75 \pm 9.99^{* *}$ & $308.51 \pm 8.32 \#$ & $200.59 \pm 8.59 \# \#$ & $280.61 \pm 11.38 \# \#$ \\
\hline \multicolumn{6}{|l|}{ LDL (mg/dl) } \\
\hline week 1- baseline (Before induction) & $48.23 \pm 3.29$ & $47.22 \pm 1.29$ & $48.01 \pm 3.31$ & $47.59 \pm 3.98$ & $47.29 \pm 3.19$ \\
\hline week 16 (Post induction) & $67.66 \pm 3.31$ & $367.61 \pm 15.22^{* *}$ & $366.21 \pm 18.30^{* *}$ & $363.57 \pm 14.69^{* *}$ & $361.63 \pm 14.17^{* *}$ \\
\hline week 20 & $68.87 \pm 3.06$ & $405.59 \pm 18.23^{* *}$ & $351.9 \pm 15.09$ & $326.36 \pm 14.09$ & $326.62 \pm 14.29$ \\
\hline week 23 & $69.72 \pm 3.25$ & $439.84 \pm 10.59^{* *}$ & $333.59 \pm 11.66$ & $271.24 \pm 11.05$ & $278.36 \pm 10.09$ \\
\hline week 26 & $73.92 \pm 2.88$ & $481.22 \pm 75.05^{* *}$ & $311.35 \pm 18.36$ & $213.59 \pm 10.29 \# \#$ & $240.65 \pm 12.07 \#$ \\
\hline \multicolumn{6}{|l|}{ VLDL (mg/dl) } \\
\hline week 1- baseline (Before induction) & $11.08 \pm 0.56$ & $11.22 \pm 0.77$ & $11.21 \pm 0.81$ & $11.10 \pm 0.67$ & $11.02 \pm 0.66$ \\
\hline week 16 (Post induction) & $14.88 \pm 0.74$ & $82.18 \pm 3.97^{* *}$ & $82.73 \pm 4.49^{* *}$ & $84.27 \pm 4.29^{* *}$ & $82.55 \pm 3.89^{* *}$ \\
\hline week 20 & $15.84 \pm 0.70$ & $90.43 \pm 3.29^{* *}$ & $77.52 \pm 4.20$ & $73.06 \pm 5.20$ & $72.38 \pm 3.34$ \\
\hline week 23 & $16.65 \pm 0.62$ & $98.68 \pm 5.02^{* *}$ & $70.31 \pm 4.35$ & $55.85 \pm 4.35 \#$ & $60.49 \pm 3.09$ \\
\hline week 26 & $16.52 \pm 0.53$ & $105.55 \pm 3.07^{* *}$ & $62.2 \pm 2.21$ & $30.64 \pm 4.04 \# \#$ & $48.51 \pm 4.52 \#$ \\
\hline \multicolumn{6}{|l|}{$\mathrm{HDL}(\mathrm{mg} / \mathrm{dl})$} \\
\hline week 1- baseline (Before induction) & $53.23 \pm 8.69$ & $53.33 \pm 7.05$ & $53.53 \pm 6.05$ & $54.59 \pm 6.29$ & $53.09 \pm 8.34$ \\
\hline week 16 (Post induction) & $69.64 \pm 3.35$ & $16.08 \pm 1.67 \$ \$$ & $15.78 \pm 2.17 \$ \$$ & $15.03 \pm 2.22 \$ \$$ & $15.03 \pm 2.19 \$ \$$ \\
\hline week 20 & $70.92 \pm 6.09$ & $13 \pm 1.38 \$ \$$ & $21.99 \pm 2.01$ & $32.18 \pm 2.56 \wedge$ & $24.28 \pm 2.65$ \\
\hline week 23 & $71.18 \pm 2.95$ & $9.32 \pm 2.08 \$ \$$ & $40.08 \pm 3.55 \wedge$ & $67.43 \pm 4.05 \wedge \wedge$ & $34.63 \pm 5.29 \wedge$ \\
\hline week 26 & $73.92 \pm 5.01$ & $5.64 \pm 2.59 \$ \$$ & $58.06 \pm 3.23 \wedge$ & $81.68 \pm 2.92 \wedge \wedge \%$ & $42.89 \pm 4.84 \wedge$ \\
\hline
\end{tabular}

**highly significant difference $(p<0.005)$ (increased values) than week 1 - baseline (Before induction) values

\# significant difference $(p<0.05)$ (decreased values) than week 16- (post induction) values

\#\# highly significant difference $(p<0.005)$ (decreased values) than week 16- (Post induction) values

$\$ \$$ highly significant difference $(p<0.005)$ (decreased values) than week 1 - baseline (Before induction) values

$\wedge$ significant difference $(p<0.05)$ (increased values) than week 16- (post induction) values

$\wedge \wedge$ highly significant difference $(\mathrm{p}<0.005)$ (increased values) than week 16 - (post induction) values

$\%$ significant difference $(p<0.05)$ (increased values) than control group in corresponding weeks

TRIG Triglycerides, TC Total cholesterol, LDL Low-density lipoproteins, VLDL Very-low-density lipoproteins, HDL High-density lipoproteins

dysfunction with reduced protein synthesis and hepatic injury. With the treatment of Mk LE, hepatic function markers were restored in a dose-dependent manner, but significantly in Mk LE-500 group $(p<0.005)$. In lovastatin $(0.5 \mathrm{mg} / \mathrm{kg} /$ day $)$ treated group few parameters improved significantly after 10 weeks of treatment $(p<$ $0.05)$.

At the end of the induction period, oxidative stress and inflammation were also significantly $(p<0.005)$ increased with RHMVO feeding, shown by elevated MDA and CRP levels. Mk LE in higher doses of $500 \mathrm{mg} / \mathrm{kg}$ was enough to restore the normal levels after 10 weeks of treatment $(p<0.005)$. Lovastatin $(0.5 \mathrm{mg} / \mathrm{kg} /$ day $)$ treated group exhibited a lesser impact on inflammatory and oxidative stress markers over 10 weeks period (Table 3).

\section{Chemical analysis of the extract}

Dry powder of curry leaves yielded 13.6\% components by weight (water-soluble). The quantitative characterization 
Table 3 Comparison of oxidative stress and liver function markers at week-26

\begin{tabular}{|c|c|c|c|c|c|}
\hline Variables & Control \#\# & $\mathrm{RHMVO}^{* *}$ & Mk LE-300 & Mk LE-500 & Lovastatin \\
\hline$\overline{C P K}(\mathrm{U} / \mathrm{L})$ & $148.17 \pm 2.29$ & $1116.85 \pm 9.27$ & $981.81 \pm 8.55^{* *}$ & $562.84 \pm 13.78 * * \#$ & $947.84 \pm 17.41^{* *} \#$ \\
\hline $\mathrm{LDH}(\mathrm{U} / \mathrm{L})$ & $212.44 \pm 45.38$ & $1214.21 \pm 9.71$ & $986.64 \pm 4.86^{* *} \#$ & $508.13 \pm 9.05 * \# \#$ & $831.42 \pm 12.85 * * \#$ \\
\hline Homocysteine $(\mu \mathrm{Mol} / \mathrm{L})$ & $4.62 \pm 0.54$ & $15.88 \pm 1.08$ & $10.57 \pm 1.33^{*} \#$ & $5.26 \pm 1.04 \# \#$ & $11.33 \pm 0.91^{* *}$ \\
\hline MDA (nMol/ml) & $8.00 \pm 1.43$ & $29.7 \pm 1.32$ & $20.68 \pm 2.42^{* *} \#$ & $12.14 \pm 1.69 * \# \#$ & $19.77 \pm 1.71 * * \#$ \\
\hline CRP (mg/dl) & $0.04 \pm 0.01$ & $14.64 \pm 2.16$ & $11.93 \pm 1.52^{* *} \#$ & $4.39 \pm 1.57 * \# \#$ & $9.81 \pm 0.98^{* *}$ \\
\hline SGPT (U/L) & $92.9 \pm 4.65$ & $376.23 \pm 8.33$ & $305.5 \pm 2.85^{* *}$ & $208.93 \pm 70.98 * \# \#$ & $301.82 \pm 10.57^{* *}$ \\
\hline SGOT (U/L) & $95.28 \pm 5.11$ & $404.28 \pm 8.77$ & $311.41 \pm 3.91^{* *}$ & $185.3 \pm 35.78$ *\#\# & $315.42 \pm 6.45^{* *}$ \\
\hline$A L P(U / L)$ & $82.89 \pm 4.8$ & $635.25 \pm 4.98$ & $556.35 \pm 3.07^{* *}$ & $217.2 \pm 9.26 * \# \#$ & $355.59 \pm 6.82^{* *} \#$ \\
\hline Total Protein (mg/dl) & $7.42 \pm 0.43$ & $2.5 \pm 0.24$ & $3.51 \pm 0.25^{*}$ & $5.45 \pm 0.29 * \#$ & $2.11 \pm 0.13^{* *}$ \\
\hline Albumin (mg/dl) & $3.42 \pm 0.46$ & $1.08 \pm 0.04$ & $2.06 \pm 0.05 \#$ & $3.09 \pm 0.12 \# \#$ & $1.56 \pm 0.43^{*}$ \\
\hline
\end{tabular}

*Significantly different from Control $(p<0.05)$. \#significantly different from RHMVO $(p<0.05)$. * Significantly different from Control $(p<0.005)$. \#\#significantly different from RHMVO $(p<0.005)$

of the chemical in Mk LE showed the significant existence of alkaloid, flavonoid, chlorophyll, polyphenol, and tannin (Table 4).

\section{Effect on atheromatous plaques area}

As shown in Fig. 2, at the end of 26 weeks RHMVO feeding resulted in extensive atheromatous plaques formation, covered about $80-85 \%$ of the aortic arch. This atheromatous plaque was noticeably attenuated by $\mathrm{Mk}$ LE in a dose-dependent manner at the end of the treatment phase. Atheromatous plaque reduced to 60 and $40 \%$ in $300 \mathrm{mg}$ and $500 \mathrm{mg}$ Mk LE doses respectively. The atheroma area was reduced by lovastatin $(0.5 \mathrm{mg} /$ $\mathrm{kg} /$ day) to $57 \%$ after 10 weeks of treatment.

\section{Hepatic histological observations}

Under the microscopic observations, widespread lipid accumulation was observed in hepatocytes in the RHMVO group (Fig. 3). The point of interest is the remarkable dose-dependent attenuation of RHMVOinduced hepatic steatosis with Mk LE treatment. The effect of Mk LE-500 is greater than the lovastatin.

\section{Discussion}

Alternative medicines are getting an important place to manage different health problems and rising as a wide field of research. Management of hyperlipidemiainduced atherosclerosis also relies on alternative medicine as an upcoming changed trend to protect and treat. Using the animal model, we created a real-life scenario and induced hyperlipidemia and atheroma formation with RHMVO and considered herbal nutritional sources to manage it.

The results of this study establish that Mk LE has the potential to treat the RHMVO-induced hyperlipidemia and atherosclerosis. The extract is found as a rich source of polyphenols, alkaloids, chlorophyll, and flavonoids. The quantitative analysis of curry leaves aqueous extract reported that there are constituents with antioxidant properties, the main reason for using curry leaves as alternative medicines and nutritional supplements in oxidative stress-induced disease studies [63-65]. Mk LE, with its immense health benefits, has been reported as a protective remedy against oxidative stress-induced diabetes [66]. The aqueous extract of curry leaves also has prolific antioxidant activities against lead and cadmiuminduced oxidative stress in animal models [66, 67]. Several in vivo $[68,69]$ and in vitro $[70,71]$ studies reported the free-radical scavenging ability and antioxidant potential of curry leaves extract concluding the immediate and strong ameliorative actions for treating oxidative stress. Considering the same facts, in this study we evaluated the dose-dependent effect of curry leaves, to treat the hyperlipidemia and oxidative stress induced by the high dose of thermally oxidized cooking oils blend. The impact was also compared with standard i.e. lovastatin

Table 4 Quantitative extraction yield and chemical content of Mk LE

\begin{tabular}{|c|c|c|c|c|c|c|}
\hline \multirow[t]{2}{*}{ Sample } & \multirow{2}{*}{$\begin{array}{l}\text { Extraction yields } \\
(\%)=\text { weight of sample } \\
\text { extract /sample } \\
\text { weight }) \times 100\end{array}$} & \multicolumn{5}{|l|}{ Chemical composition } \\
\hline & & $\begin{array}{l}\text { Total phenols (mg } \\
\text { gallic acid equivalent } \\
\text { /g Mk LE) }\end{array}$ & $\begin{array}{l}\text { Total flavonoid (mg } \\
\text { catechin equivalent. } \\
\text { /g Mk LE) }\end{array}$ & $\begin{array}{l}\text { Chlorophyll } \\
\text { content (mg/ } \\
\text { g Mk LE }\end{array}$ & $\begin{array}{l}\text { Alkaloid content } \\
\text { (mg bismuth } \\
\text { nitrate/g Mk LE) }\end{array}$ & $\begin{array}{l}\text { Total tannin (mg } \\
\text { tannic acid } \\
\text { equivalent /g Mk LE) }\end{array}$ \\
\hline $\begin{array}{l}\text { M.koenigii leaf } \\
\text { aqueous extract } \\
\text { (Mk LE) }\end{array}$ & $15.63 \pm 0.46$ & $46.78 \pm 0.07$ & $6.1 \pm 0.36$ & $0.40 \pm 0.009$ & $37.9 \pm 0.28$ & $0.129 \pm 0.008$ \\
\hline
\end{tabular}



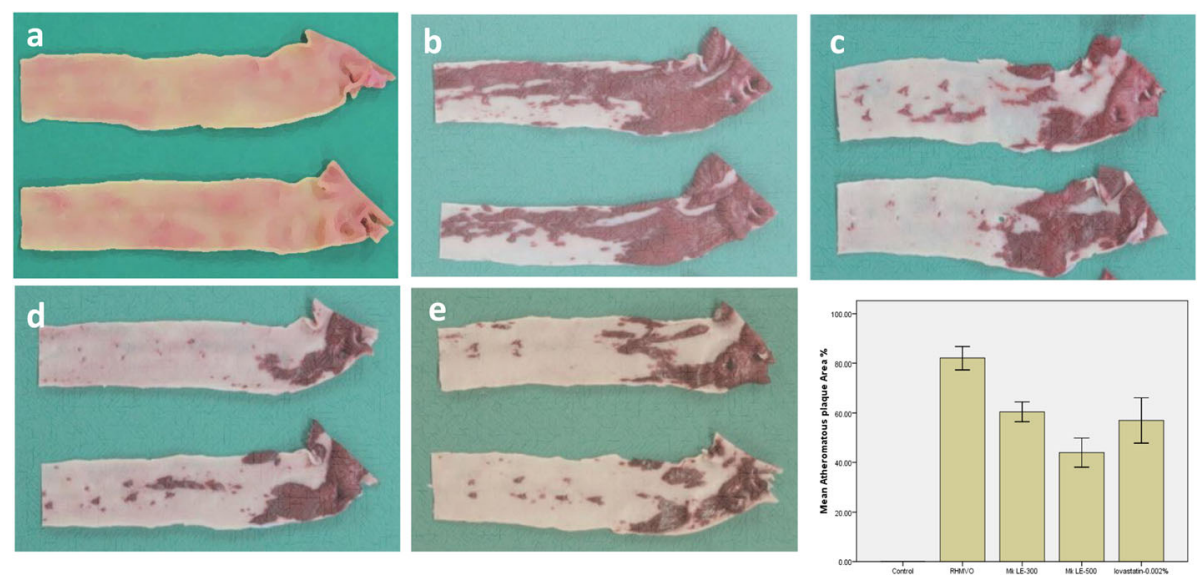

Fig. 2 Atheroma formation and resolution at the end of 26 weeks: (a) Control; with no atheromatous plaques formation (b) RHMVO fed group; extensive atheromatous plaques formation, covered about 80-85\% of the aortic arch (c) Mk LE-300 group; atheromatous plaque reduced to 60\% (d) Mk LE-500 group; atheromatous plaque reduced to 40\% (e) lovastatin group; Atheromatous plaque reduced to $57 \%$

from the statin group. Mk LE at a dose of $500 \mathrm{mg} / \mathrm{kg}$ was found effective in a shorter period, to treat oxidative damage and hyperlipidemia mediated by RHMVO compared to $300 \mathrm{mg} / \mathrm{kg}$. The histopathological and macroscopic results showed that to treat RHMVO-induced atherosclerosis the effect of MK LE in $500 \mathrm{mg} / \mathrm{kg}$ of body weight is comparable with lovastatin, as all the study parameters were significantly reduced in the Mk LE-500 group.

Thermally oxidized oil is the most significant source of oxidative damage for human health if used daily for a long time. In RHMVO fed groups, highly elevated levels

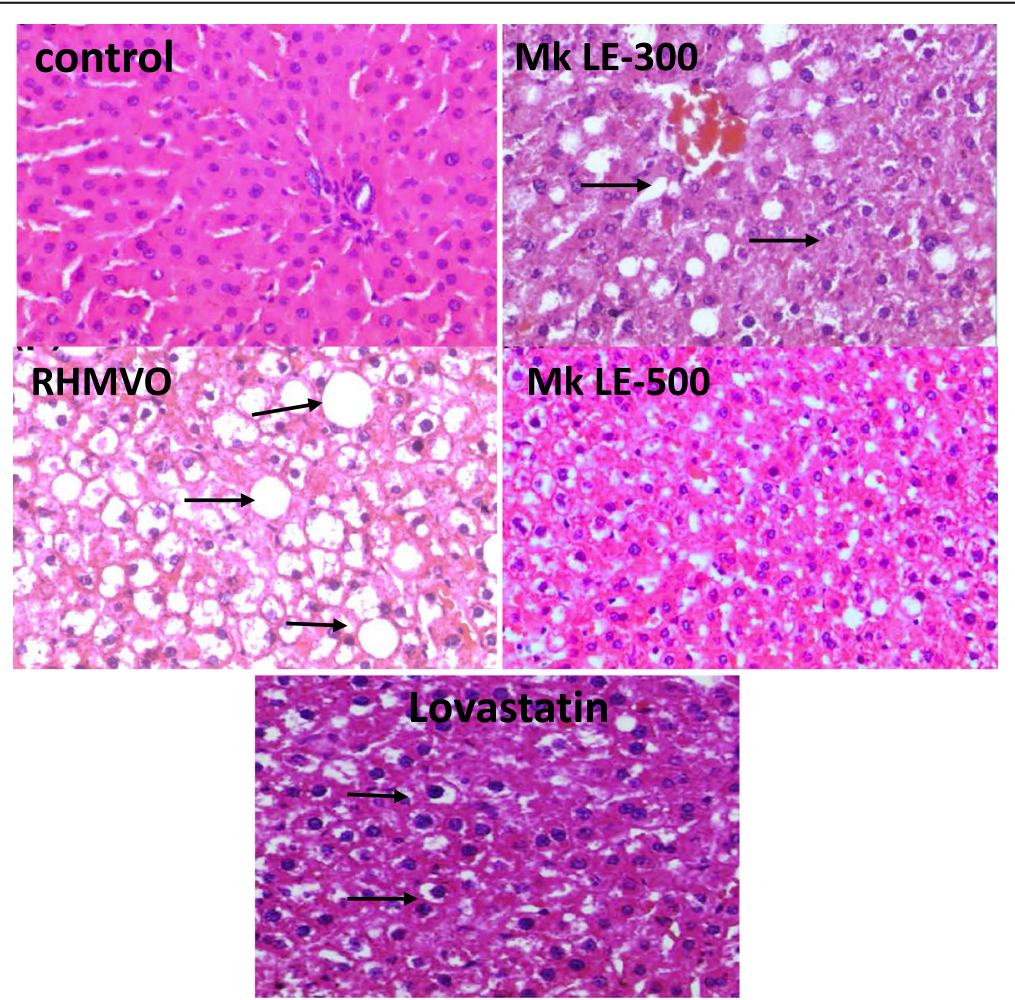

Fig. 3 Histopathological finding of rabbits' liver after 10 weeks of treatment. Control: a microscopic view of a normal liver. RHMVO fed group: a microscopic view of fatty liver showing distended hepatocytes due to fat vacuoles. Mk LE-300 group: observed a lesser number of fat vacuoles in the portal area. Mk LE-500 group: observed an insignificant number of fat vacuoles. Lovastatin group: observed an insignificant number of fat vacuoles (200x magnification) 
of SGOT (extremely sensitive and a precise biomarker for hepatotoxicity) and ALP (a main biomarker of hepatic and biliary defects like cholestasis) [72] reflected hepatocytes damage and tissue interruption, allowing the leakage of intracellular enzymes in blood [73]. The liver is damaged by excessive free radicals and may result in hepatitis, cirrhosis, and hepatic tumor [74]. Consistent with the diminished serum albumin and total protein levels and supported by the significantly enlarged liver and histopathological changes in hepatocytes of RHMVO fed rabbits. The results of our study are consistent with the previous study, reported significantly reduced serum SGPT and SGOT levels in curry leaves pretreated groups against the lead-induced model, a clear indication of hepatic protection [75, 76], but the maximum effective dose of curry leaves was $100 \mathrm{mg} / \mathrm{kg}$ body weight.

The noticeable presence of carbazole alkaloids and tannins in Mk LE analysis exhibit excellent hepatoprotective activity through their anti-lipid peroxidation and antioxidant potential [77]. In the present study, Mk LE considerably inhibited fat accumulation in hepatocytes and the formation of hypercholesterolemia-induced atheroma. Besides, curry leaves extract attenuated lipid peroxidation and oxidative membrane injury in the organs showing lipidosis. Statins are preferentially prescribed to improve blood lipid profile through suppressing hepatic cholesterol synthesis [11]. However, it's reported in previous studies that long-term use and over-dosage of the statins results in severe hepatotoxicity, whereas low doses failed to control blood cholesterol levels effectively from the diet [10-12]. Statins are reported to produce adverse effects in up to $33 \%$ of patients [78]. Notably, the management of cerebrovascular and cardiovascular disease risk factors in preventive mode is more effective than the therapeutic mode, as after the outbreak of these diseases time to death is very short [11]. Therefore, it has been recommended to follow dietary restrictions and choose appropriate oils and fats. In addition, the excessive consumption of oils and fats especially in thermally oxidized form is also known to cause vascular diseases, such as reduced vasorelaxation responses, endothelial malfunction [16], lipid peroxidation [17], atherosclerosis, and oxidative stress [19].

Reactive oxygen species (ROS) are free radicals with one or more unpaired electrons. Major ROS include superoxide anion, hydrogen peroxide, and the hydroxyl radical [79]. In addition to ROS, reactive nitrogen species (RNS), including peroxynitrite (NO3-), nitric oxide radical (NO), and S-nitrosothiols, also contribute to the generation of oxidative stress. ROS are often generated as byproducts of cellular metabolic reactions and exogenous induction. These ROS produce homeostatic imbalances, and overwhelm cellular antioxidants defense system and results in oxidative stress, that further damage biomolecules such as proteins, nucleic acids, and lipids which eventually, induces cell death and tissue injury [80, 81]. Subsequently promotes the development of age-dependent diseases, like cancer, atherosclerosis, arthritis, etc. [82]. MK LE has several potent antioxidants, such as isomahanine, isolongifolene, mahanine, mahanimbine, koenimbine,girinimbine, koenoline, and O-methylmurrayamine [83, 84]. These antioxidants exhibit 2,2-diphenyl-1-picrylhydrazyl (DPPH) free radical scavenging activity, inhibition of NO radical, and thiobarbituric acid reactive substances (TBARS) activity $[85,86]$.

Mitochondria are the powerhouse of the living cell and play a vital role in scavenging free radicals and controlling programmed cell death and/or the apoptosissignaling pathway [87]. RHMVO-induced mitochondrial damage results in decreased adenosine triphosphate (ATP) production, increased ROS generation, impaired calcium buffering, damage to mitochondrial DNA (mtDNA). An altered mitochondrial morphology and alterations in mitochondrial fission and fusion. Mitochondrial dysfunction is characterized by a loss of efficiency in the electron transport chain, as well as reductions in the synthesis of high-energy molecules [88]. Mitochondrial dysfunction is a characteristic of all chronic diseases including neurodegenerative disorders, CVDs, diabetes, auto-immune diseases, atherosclerosis, and others $[89,90]$.

Recent studies have evaluated the neuroprotective activities of compounds isolated from MK LE. A study reported that the bioactive compounds present in MK LE exhibited the ability to repair the mitochondrial damage and restore the mitochondrial membrane potential levels [91-93]. Previous findings have demonstrated that MK LE and its primary active compounds regulate multiple signaling pathways, including phosphatidylinositol 3 kinase (PI3K)/protein kinase $\mathrm{B}$ (AKT), mammalian target of rapamycin (mTOR) and mitogen-activated protein kinase (MAPK). M. koenigii and its primary active compounds exert complementary effects on oxidative stress and the alteration of proteins [94, 95].

The lipid-lowering activity of MK LE is reported in several animal studies, in the different doses, in the range of $80-600 \mathrm{mg}[20,21,23]$. To our knowledge, this is the first animal study that evaluated the effect of MK $\mathrm{LE}$, in RHMVO-induced hypercholesterolemia in animals, also using the standard (lovastatin). In the dose of $500 \mathrm{mg} / \mathrm{kg}$ MK LE significantly reduced the TG, total cholesterol, LDL, and VLDL, also, increased HDL levels and reduced the cardiac infarction risk through the recovery of the arterial endothelial cells functions [96]. Better response with high doses is also supported by other studies [20, 97]. The requirement of a longer 
duration to treat dyslipidemia is also supported by a previous study [97].

The high value of CRP is possibly indicative of developing cardiovascular disease, active inflammation, bacterial infection [98], and inflammatory bowel disease, and other similar conditions like intestinal lymphoma and tuberculosis [99]. Overexpression of CPK, homocysteine, and LDH has been linked with the number of disorders including tissue damages, ROS induced inflammation, and endothelial cell injury [100]. The elevated levels of all these tissues damaging markers are suggestive of increased risk of hypertension, diabetes, and CVDs $[101,102]$. Reduction in these parameters along with reduced oxidative stress is an additional benefit of MK LE, compared to statins.

Moreover, it was demonstrated that MK LE inhibits platelet aggregation when given with coriander extract [23], thus further explains the MK LE potential to treat RHMVO-induced atherosclerosis. Formation of thrombus and atherosclerosis takes place when vascular endothelial walls get injured during oxidized LDLmediated oxidative reactions and activated platelets get attached to this area. Therefore, curry leaves extract can be the alternate option for investigators to treat hyperlipidemia, oxidative stress, and atherosclerosis medicated by consumption of a higher amount of RHMVO in daily life.

\section{Conclusion}

The results of this study conclude that Mk LE exhibits potent antioxidant and antidyslipidemic properties that can be used to treat RHMVO-induced hyperlipidemia and atherosclerosis in optimal doses and duration. Over the past few decades, the prevalence of coronary artery diseases has increased significantly in South Asians and predicted to be more than 24 million in 2035. Curry leaves are basically of south Asian origin, so it can be the better, easier, and cheaper option as nutrient supplement and remedy.

\section{Abbreviations \\ LDL: Low-density lipoproteins; VLDL: Very-low-density lipoproteins; TG: Triglycerides; TC: Total cholesterol; HDL: High-density lipoproteins: CVDs: Cardiovascular diseases; HMG-CoA: 3-hydroxy-3- methylglutanyl- coenzyme A; ROS: Reactive oxygen species; MDA: Malondialdehyde; CPK: Creatine phosphokinase; LDH: Lactate dehydrogenase; CRP: C-reactive protein}

\section{Acknowledgments}

We acknowledge Abdul Moiz Hussain and Dr. Junaid lqbal for providing the technical and editing support in the process of final submission. Authors are highly thankful to Dr. Muhammad Mohtasheemul Hasan of the Department of Pharmacognosy, the University of Karachi for providing specimen authentication for plant material and oils, used in the study.

\section{Disclosure statement}

The authors of this article do not have any commercial or financial conflicts of interest.

\section{Authors' contributions}

GA: Substantial contributions to the conception or design of the work. Performed the study and wrote the paper, Analysis, or interpretation of data. AS: Revised the work critically for important intellectual content. Supervised the research, Final approval of the version published. KH: co-wrote the paper and final review of the manuscript. ASH: co-wrote the manuscript in revision phase with Pl, edited the paper as per reviewers' comments. ZA: co-wrote the manuscript in revision phase with $\mathrm{Pl}$, edited the paper as per reviewers' comments. The authors read and approved the final manuscript before submission.

\section{Funding}

No source of funding in the research.

\section{Availability of data and materials}

The datasets used and/or analyzed during the current study are available from the corresponding author on reasonable request.

\section{Ethics approval and consent to participate}

Research study protocol along with the procedures to be followed during our research study, euthanasia, and ethical authorization of the study all got approved from the Board of Advanced Studies \& Research (BASR) of the University of Karachi (BASR number:03296/Pharm).

\section{Consent for publication}

Not applicable.

\section{Competing interests}

The authors declare that they have no competing interests" in this section.

\section{Author details}

'Department of Pharmacology, Faculty of Pharmacy and Pharmaceutical Sciences, University of Karachi, Karachi, Pakistan. ${ }^{2}$ Department of Pharmacy, Aga Khan University Hospital, Stadium Road (Main Pharmacy), P.O Box 3500, Karachi 74800, Pakistan. ${ }^{3}$ Institute of Pharmaceutical Sciences, Peoples University of Medical and Health Sciences, Nawabshah, Sindh, Pakistan.

Received: 12 October 2019 Accepted: 2 July 2020

Published online: 14 July 2020

\section{References}

1. Alberti G. Introduction to the metabolic syndrome. Eur Heart J Supplem. 2005;7(suppl_D):D3-5.

2. Alberti KGM, Zimmet P, Shaw J. The metabolic syndrome-a new worldwide definition. Lancet. 2005;366(9491):1059-62.

3. Grundy SM, et al. Diagnosis and management of the metabolic syndrome: an American Heart Association/National Heart, Lung, and Blood Institute scientific statement. Circulation. 2005;112(17):2735-52.

4. Reaven GM. Role of insulin resistance in human disease. Diabetes. 1988 ; 37(12):1595-607.

5. Manninen $V$, et al. Joint effects of serum triglyceride and LDL cholesterol and HDL cholesterol concentrations on coronary heart disease risk in the Helsinki heart study. Implications for treatment. Circulation. 1992:85(1):37-45.

6. Arca $M$, et al. Usefulness of atherogenic dyslipidemia for predicting cardiovascular risk in patients with angiographically defined coronary artery disease. Am J Cardiol. 2007;100(10):1511-6.

7. Linton MF, et al. The role of lipids and lipoproteins in atherosclerosis. In: Endotext; 2019. MDText. com, Inc.

8. Blum A, Simsolo C, Hasin Y. 3-Hydroxy-3-methylglutaryl coenzyme a (HMG$\mathrm{CoA}$ ) reductase inhibitors (statins), atherosclerosis and coronary syndromes. Atherosclerosis. 2004;175(1):1-5.

9. Larsen ML, Illingworth DR. Drug treatment of dyslipoproteinemia. Med Clin N Am. 1994;78(1):225-45.

10. Davis SM, Donnan GA. Secondary prevention after ischemic stroke or transient ischemic attack. N Engl J Med. 2012;366(20):1914-22.

11. Kernan $W N$, et al. Guidelines for the prevention of stroke in patients with stroke and transient ischemic attack: a guideline for healthcare professionals from the American Heart Association/American Stroke Association. Stroke. 2014;45(7):2160-236. 
12. Collins R, et al. Interpretation of the evidence for the efficacy and safety of statin therapy. Lancet. 2016;388(10059):2532-61.

13. Andrikopoulos NK, et al. Performance of virgin olive oil and vegetable shortening during domestic deep-frying and pan-frying of potatoes. Int J Food Sci Technol. 2002;37(2):177-90.

14. Sommerfeld M. Trans unsaturated fatty acids in natural products and processed foods. Prog Lipid Res. 1983;22(3):221-33.

15. Owu D, Orie N, Osim E. Altered responses of isolated aortic smooth muscle following chronic ingestion of palm oil diets in rats. Afr J Med Med Sci. 1997;26:83-6.

16. Lopez-Garcia E, et al. Consumption of trans fatty acids is related to plasma biomarkers of inflammation and endothelial dysfunction. J Nutr. 2005;135(3): 562-6.

17. Adam SK, et al. Consumption of repeatedly heated soy oil increases the serum parameters related to atherosclerosis in ovariectomized rats. Tohoku J Exp Med. 2008;215(3):219-26.

18. Adam SK, et al. Effects of repeatedly heated palm oil on serum lipid profile, lipid peroxidation and homocysteine levels in a post-menopausal rat model. McGill J Med: MJM. 2008;11(2):145.

19. Leong XF, et al. Heated palm oil causes rise in blood pressure and cardiac changes in heart muscle in experimental rats. Arch Med Res. 2008;39(6): $567-72$

20. Kesari AN, et al. Studies on the glycemic and lipidemic effect of Murraya koenigii in experimental animals. J Ethnopharmacol. 2007;112(2):305-11.

21. Tembhurne SV, Sakarkar DM. Anti-obesity and hypoglycemic effect of ethanolic extract of Murraya koenigii (L) leaves in high fatty diet rats. Asian Pacific J Trop Dis. 2012;2:S166-8.

22. Molly J, et al. Curry leaf powder and cucumber slices attenuate hyperlipidemia in post menopausal women: a randomized control single blinded study. Int J Pharm Sci Res. 2017:8(4):1858.

23. Suneetha WJ, Krishnakantha T. Antiplatelet activity of coriander and curry leaf spices. Pharm Biol. 2005;43(3):230-3.

24. Firdaus SB, et al. Protective effect of aqueous leaf extract of Murraya koenigi against lead induced oxidative stress in rat liver, heart and kidney: a dose response study. Asian J Pharm Clin Res. 2012;5(4):54-8.

25. Kesari AN, Gupta RK, Watal G. Hypoglycemic effects of Murraya koenigii on normal and alloxan-diabetic rabbits. J Ethnopharmacol. 2005;97(2):247-51.

26. Debosree $\mathrm{G}$, et al. Protective effect of aqueous leaf extract of Murraya koenigi against lead induced oxidative stress in rat liver, heart and kidney: a dose response study. Asian J Pharm Clin Res. 2012;5:54-8.

27. Sun $Y-Z$, et al. Anti-atherosclerotic effect of hesperidin in LDLr-/- mice and its possible mechanism. Eur J Pharmacol. 2017;815:109-17.

28. Liu C, et al. Apoc2 loss-of-function zebrafish mutant as a genetic model of hyperlipidemia. Dis Model Mech. 2015;8(8):989-98.

29. Im KH, et al. Hyperlipidemic inhibitory effects of Phellinus pini in rats fed with a high fat and cholesterol diet. Mycobiology. 2018;46(2):159-67.

30. Sithu SD, et al. Atherogenesis and metabolic dysregulation in LDL receptorknockout rats. JCl insight. 2017;2(9):42-45.

31. Huang L, et al. CRISPR/Cas9-mediated ApoE-/-and LDLR-/-double gene knockout in pigs elevates serum LDL-C and TC levels. Oncotarget. 2017; 8(23):37751.

32. Mapara M, Thomas BS, Bhat K. Rabbit as an animal model for experimental research. Dental Res J. 2012:9(1):111.

33. Fan J, et al. Rabbit models for the study of human atherosclerosis: from pathophysiological mechanisms to translational medicine. Pharmacol Ther. 2015;146:104-19

34. Pogwizd SM, Bers DM. Rabbit models of heart disease. Drug Discov Today Dis Model. 2008;5(3):185-93.

35. Shukla R, et al. Hypolipidemic effect of water extract officus bengalensis in alloxan induced diabetes mellitus in rabbits. Indian J Clin Biochem. 1995; 10(2):119.

36. Akhtar MS, lqbal J. Evaluation of the hypoglycaemic effect of Achyranthes aspera in normal and alloxan-diabetic rabbits. J Ethnopharmacol. 1991;31(1): 49-57.

37. Akhtar M, Khan M, Malik M. Hypoglycaemic activity of Alpinia galanga rhizome and its extracts in rabbits. Fitoterapia. 2002;73(7-8):623-628.

38. Del Carmen Flores-Álvarez $\mathrm{M}$, et al. The effect of food type (fish nuggets or French fries) on oil blend degradation during repeated frying. J Food Sci. 2012;77(11):C1136-43.

39. Katragadda HR, et al. Emissions of volatile aldehydes from heated cooking oils. Food Chem. 2010;120(1):59-65.
40. Basuny $A$, et al. Production of mixture more resistance from some vegetable oils for frying process. MOJ Food Process Technol. 2015;1(4):121-7.

41. Aydar AY, Rodriguez-Martinez V, Farkas BE. Determination and modeling of contact angle of canola oil and olive oil on a PTFE surface at elevated temperatures using air or steam as surrounding media. LWT-Food Sci Technol. 2016;65:304-10.

42. Fauziah A, Razali I, Nor-Aini S. Frying performance of palm olein and high oleic sunflower oil during batch frying of potato crisps. Palm Oil Developments. 2000;3:1-7.

43. Siddiq A, et al. Oxidative stress and lipid per-oxidation with repeatedly heated mix vegetable oils in different doses in comparison with single time heated vegetable oils. Pak J Pharm Sci. 2019;32(5):51-56.

44. Mahmoodzadeh S, Fliegner D, Dworatzek E. Sex differences in animal models for cardiovascular diseases and the role of estrogen. In: Sex and Gender Differences in Pharmacology: Springer; 2013. p. 23-48.

45. Regitz-Zagrosek $V$. Therapeutic implications of the gender-specific aspects of cardiovascular disease. Nat Rev Drug Discov. 2006;5(5): 425-39.

46. Sarfraz M, Sajid S, Ashraf MA. Prevalence and pattern of dyslipidemia in hyperglycemic patients and its associated factors among Pakistani population. Saudi J Biological Sci. 2016;23(6):761-6.

47. Zaid M, Hasnain S. Plasma lipid abnormalities in Pakistani population: trends, associated factors, and clinical implications. Braz J Med Biol Res. 2018;51(9): 24.

48. Mclver LA, Siddique MS. Atorvastatin. In: StatPearls. Treasure Island: StatPearls Publishing; 2019.

49. Laber-Laird K, Swindle MM, Flecknell P. Handbook of rodent and rabbit medicine. Amsterdam: Pergamon/Elsevier Science Ltd; 1996.

50. Awad $\mathrm{K}$, et al. Effects of morning vs evening statin administration on lipid profile: a systematic review and meta-analysis. J Clin Lipidol. 2017;11(4):97285 e9.

51. Awad K, Banach M. The optimal time of day for statin administration: a review of current evidence. Curr Opin Lipidol. 2018;29(4):340-5.

52. Underwood W, Anthony R. AVMA Guidelines for the Euthanasia of Animals: 2020 edition; 2013.

53. Kikugawa $\mathrm{K}$, et al. Interpretation of the thiobarbituric acid reactivity of rat liver and brain homogenates in the presence of ferric ion and ethylenediaminetetraacetic acid. Anal Biochem. 1992;202(2):249-55.

54. Hamsi MA, et al. Effect of consumption of fresh and heated virgin coconut oil on the blood pressure and inflammatory biomarkers: an experimental study in Sprague Dawley rats. Alexandria J Med. 2015;51(1):53-63.

55. Sheu WH-H, Lee W-J, Chen Y-T. Plasma homocysteine concentrations and insulin sensitivity in hypertensive subjects. Am J Hypertens. 2000;13(1):14-20.

56. Cheong $\mathrm{SH}$, et al. Spirulina prevents atherosclerosis by reducing hypercholesterolemia in rabbits fed a high-cholesterol diet. J Nutr Sci Vitaminol. 2010;56(1):34-40.

57. Park D, et al. Anti-hypercholesterolemic and anti-atherosclerotic effects of polarized-light therapy in rabbits fed a high-cholesterol diet. Laboratory Anim Res. 2012:28(1):39-46.

58. Jang JY, et al. An ethanolic extract of Angelica gigas improves atherosclerosis by inhibiting vascular smooth muscle cell proliferation Laboratory Anim Res. 2014;30(2):84-9.

59. Sefi M, et al. Mitigating effects of antioxidant properties of Artemisia campestris leaf extract on hyperlipidemia, advanced glycation end products and oxidative stress in alloxan-induced diabetic rats. Food Chem Toxicol. 2010;48(7):1986-93.

60. Sreevidya N, Mehrotra S. Spectrophotometric method for estimation of alkaloids precipitable with Dragendorff's reagent in plant materials. J AOAC Int. 2003;86(6):1124-7.

61. Polshettiwar S, et al. Spectrophotometric estimation of total tannins in some ayurvedic eye drops. Indian J Pharm Sci. 2007;69(4):574.

62. Kizhedath A, Suneetha V. Estimation of chlorophyll content in common household medicinal leaves and their utilization to avail health benefits of chlorophyll. J Pharm Res. 2011;4(5):1412-3.

63. Tachibana $Y$, et al. Antioxidative activity of carbazoles from Murraya koenigii leaves. J Agric Food Chem. 2001;49(11):5589-94.

64. Ningappa MB, Dinesha R, Srinivas L. Antioxidant and free radical scavenging activities of polyphenol-enriched curry leaf (Murraya koenigii L.) extracts. Food Chem. 2008;106(2):720-8.

65. Santos CCdMP, et al. Antinociceptive and antioxidant activities of phytol in vivo and in vitro models. Neuroscience J. 2013;2013:40-49. 
66. Arulselvan P, Subramanian SP. Beneficial effects of Murraya koenigii leaves on antioxidant defense system and ultra structural changes of pancreatic $\beta$-cells in experimental diabetes in rats. Chem Biol Interact. 2007;165(2):155-64.

67. Ghosh D, et al. Hepatoprotective activity of aqueous leaf extract of Murraya koenigii against lead-induced hepatotoxicity in male wistar rat. Int J Pharm Pharm Sci. 2013;5(1):285-95.

68. Khan BA, Abraham A, Leelamma S. Biochemical response in rats to the addition of curry leaf (Murraya koenigii) and mustard seeds (Brassica juncea) to the diet. Plant Foods Hum Nutr. 1996;49(4):295-9.

69. Iyer $U$, Mani U. Studies on the effect of curry leaves supplementation (Murraya koenigi) on lipid profile, glycated proteins and amino acids in non-insulin-dependent diabetic patients. Plant Foods Hum Nutr. 1990; 40(4):275-82.

70. Rahman MM, Gray Al. A benzoisofuranone derivative and carbazole alkaloids from Murraya koenigii and their antimicrobial activity. Phytochemistry. 2005; 66(13):1601-6.

71. Roy MK, et al. Mechanism of mahanine-induced apoptosis in human leukemia cells (HL-60). Biochem Pharmacol. 2004;67(1):41-51.

72. Schefer $\mathrm{K}$, et al. Laboratory, electrocardiographic, and echocardiographic detection of myocardial damage and dysfunction in an Arabian mare with nutritional masseter myodegeneration. J Vet Intern Med. 2011;25(5):1171-80.

73. Quiles $\mathrm{J}$, et al. The intake of fried virgin olive or sunflower oils differentially induces oxidative stress in rat liver microsomes. Br J Nutr. 2002;88(1):57-65.

74. Ilavenil S, et al. Hepatoprotective mechanism of lycorine against carbon tetrachloride induced toxicity in Swiss albino mice-a proteomic approach. Asian Pacific J Reprod. 2015;4(2):123-8.

75. Gutteridge JM, Halliwell B. Free radicals and antioxidants in the year 2000: a historical look to the future. Ann N Y Acad Sci. 2000:899(1):136-47.

76. Niu S-L, Mitchell DC, Litman BJ. Trans fatty acid derived phospholipids show increased membrane cholesterol and reduced receptor activation as compared to their cis analogs. Biochemistry. 2005;44(11):4458-65.

77. Sathaye $\mathrm{S}$, et al. Hepatoprotective effects of aqueous leaf extract and crude isolates of Murraya koenigii against in vitro ethanol-induced hepatotoxicity model. Exp Toxicol Pathol. 2011;63(6):587-91.

78. Bays H. Statin safety: an overview and assessment of the data-2005. Am J Cardiol. 2006:97(8):S6-S26.

79. Dexter $D$, et al. Increased nigral iron content and alterations in other metal ions occurring in brain in Parkinson's disease. J Neurochem. 1989;52(6): 1830-6.

80. Brand MD, et al. Mitochondrial superoxide: production, biological effects, and activation of uncoupling proteins. Free Radic Biol Med. 2004;37(6):755-67

81. Thannickal VJ, Fanburg BL. Reactive oxygen species in cell signaling. Am J Physiol Lung Cell Mol Physiol. 2000;279(6):L1005-28.

82. Hoidal JR. Reactive oxygen species and cell signaling. Am J Respir Cell Mol Biol. 2001;25(6):661-3.

83. Gill NS, Sharma B. Study on antioxidant potential of Murraya koenigii leaves in Wistar rats. Pak J Biol Sci. 2014;17(1):126-9.

84. Rehana D, et al. In vitro antioxidant and antidiabetic activities of zinc oxide nanoparticles synthesized using different plant extracts. Bioprocess Biosyst Eng. 2017;40(6):943-57

85. Kusuma IW, et al. Biological activity and phytochemical analysis of three Indonesian medicinal plants, Murraya koenigii, Syzygium polyanthum and Zingiber purpurea. J Acupunct Meridian Stud. 2011;4(1):75-9.

86. Tomar RS, Banerjee S, Kaushik S. Assessment of antioxidant activity of leaves of Murraya koenigii extracts and it's comparative efficacy analysis in different solvents. Pharm Sci Res. 2017;9(3):7-11.

87. Harman D. The biologic clock: the mitochondria? J Am Geriatr Soc. 1972; 20(4):145-7.

88. Kushnareva Y, Murphy AN, Andreyev A. Complex I-mediated reactive oxygen species generation: modulation by cytochrome $C$ and NAD $(P)+$ oxidation-reduction state. Biochem J. 2002;368(2):545-53.

89. Ischiropoulos $\mathrm{H}$, Beckman JS. Oxidative stress and nitration in neurodegeneration: cause, effect, or association? J Clin Invest. 2003;111(2): 163-9.

90. Anderson $\mathrm{S}$, et al. Sequence and organization of the human mitochondrial genome. Nature. 1981;290(5806):457-65.

91. Iman $\mathrm{V}$, et al. Anticancer and anti-inflammatory activities of girinimbine isolated from Murraya koenigii. Drug Des Devel Ther. 2017;11:103.

92. Arun A, et al. Anti-colon cancer activity of Murraya koenigii leaves is due to constituent murrayazoline and O-methylmurrayamine a induced mTOR/AKT downregulation and mitochondrial apoptosis. Biomed Pharmacother. 2017; 93:510-21.

93. Balakrishnan $\mathrm{R}$, et al. Isolongifolene attenuates rotenone-induced mitochondrial dysfunction, oxidative stress and apoptosis. Front Biosci (Schol Ed). 2018;10:248-61.

94. Chen M, et al. Mahanine induces apoptosis, cell cycle arrest, inhibition of cell migration, invasion and PI3K/AKT/mTOR signalling pathway in glioma cells and inhibits tumor growth in vivo. Chem Biol Interact. 2019;299:1-7.

95. Yu Y, et al. Globularifolin exerts anticancer effects on glioma U87 cells through inhibition of Akt/mTOR and MEK/ERK signaling pathways in vitro and inhibits tumor growth in vivo. Biochimie. 2017;142:144-51.

96. Eussen SR, et al. Effects of n-3 fatty acids on major cardiovascular events in statin users and non-users with a history of myocardial infarction. Eur Heart J. 2012;33(13):1582-8.

97. Vinuthan $\mathrm{M}$, et al. Changes in blood lipid profile after administration of Murraya Koenigii spreng (curry leaf) extracts in Normal Sprague Dawley rats. Indian J Anim Res. 2007:41(3):223-5.

98. Chew KS. What's new in emergencies trauma and shock? C-reactive protein as a potential clinical biomarker for influenza infection: more questions than answers. J Emerg Trauma Shock. 2012;5(2):115.

99. Liu S, et al. Preliminary case-control study to evaluate diagnostic values of C-reactive protein and erythrocyte sedimentation rate in differentiating active Crohn's disease from intestinal lymphoma, intestinal tuberculosis and Behcet's syndrome. Am J Med Sci. 2013;346(6):467-72.

100. Drent $M$, et al. Usefulness of lactate dehydrogenase and its isoenzymes as indicators of lung damage or inflammation. Eur Respir J. 1996;9(8):1736-42.

101. Dehghan A, et al. Genetic variation, C-reactive protein levels, and incidence of diabetes. Diabetes. 2007:56(3):872-8.

102. Pradhan $A D$, et al. C-reactive protein, interleukin 6 , and risk of developing type 2 diabetes mellitus. Jama. 2001;286(3):327-34

\section{Publisher's Note}

Springer Nature remains neutral with regard to jurisdictional claims in published maps and institutional affiliations.
Ready to submit your research? Choose BMC and benefit from:

- fast, convenient online submission

- thorough peer review by experienced researchers in your field

- rapid publication on acceptance

- support for research data, including large and complex data types

- gold Open Access which fosters wider collaboration and increased citations

- maximum visibility for your research: over $100 \mathrm{M}$ website views per year

At $\mathrm{BMC}$, research is always in progress.

Learn more biomedcentral.com/submissions 